Nicolas Chambon

Docteur en sociologie

Equipe «politiques de la connaissance », Centre Max Weber (UMR 5283 CNRS)

ANR-REMILAS

Orspere-Samdarra (CH Le Vinatier)

Nicolas.chambon@cnrs.fr

\title{
Nicolas.chambon@ch-le-vinatier.fr
}

Thème de recherche : Migration, précarité, santé mentale, mobilisation

Mots clés de l'article : récit, sans-papiers, migrant, demandeur d'asile, personne, narrativité

\section{Raconter son histoire comme personne Les migrants et leurs récits}

La plupart des migrants primo-arrivants sur le territoire français (hors demande de regroupement familial ou titre de séjour étudiant) sollicite aujourd'hui la demande d'asile. En 2017, ce sont plus de 100000 personnes qui ont eu recours à cette procédure. Elle est régie par la convention de Genève entrée en vigueur en 1954 et est destinée à la personne qui : «craignant avec raison d'être persécutée du fait de sa race, de sa religion, de sa nationalité, de son appartenance à un certain groupe social ou de ses opinions politiques, se trouve hors du pays dont elle a la nationalité et qui ne peut ou, du fait de cette crainte, ne veut se réclamer de la protection de ce pays ». Le requérant, après son passage à la PADA (Plateforme d'Accueil des Demandeurs d'Asile) se rend au GUDA (Guichet Unique de la Demande d'Asile) pour déposer sa demande de protection. On lui remet alors un dossier OFPRA (l'Office Français Pour les Réfugiés et les Apatrides) : il a 21 jours pour envoyer son récit rédigé en français. Le dossier comprend deux parties, l'une porte sur l'état civil et l'autre une sur la nature et le motif des persécutions ou menaces que le demandeur d'asile a subi, ainsi que les raisons qui l'empêche d'être protégé par son État. Ce récit est d'abord examiné par un agent de l'OFPRA en présence du demandeur et si besoin d'un interprète. Le requérant a aussi la possibilité d'inviter un avocat ou un représentant d'une association habilitée. Si la personne est déboutée de sa demande, elle peut faire un recours devant la CNDA (Cour 
Nationale du Demandeur d'Asile), généralement avec des preuves supplémentaires et à l'appui d'un récit en partie dissemblable.

Nous soutenons ici que cette contrainte administrative de devoir se raconter est une épreuve pour le requérant, qui doit se raconter comme «personne d'autre » tout en devant respecter un format d'expression du récit, notamment auprès de ses potentiels soutiens. Cette proposition prend appui sur une thèse ${ }^{1}$ dont l'enquête portait les modalités de soutien aux migrants en situation irrégulière. Nous nous intéressons ici aux conséquences de l'injonction à cette mise en récit sur les requérants et ce que peut signifier que s'exposer comme « personne » à part entière.

\section{Injonction biographique et récit formaté}

Le demandeur d'asile est généralement accompagné par des intervenants sociaux pour produire le récit dans le cadre de procédures, intervenants qui ont la connaissance du format et des saillances attendues dans l'histoire de la personne requérante qu'il importe de faire valoir. Ce récit est alors formaté pour les administrations (Franguiadakis, Jaillardon, Belkis, 2004, 132-137) et ne laisse pas de place pour une autre écriture de l'histoire de vie. Il importe en effet pour les intervenants sociaux d'accompagner cette demande, d'offrir les conditions optimales permettant l'obtention de l'asile, par un suivi administratif, social et sanitaire. Concrètement cela passe par l'aide à la rédaction du récit, l'explication des démarches, l'aide aux recours... L'individu est alors « cadré » sous un angle administratif.

Le récit porte l'empreinte de la procédure. C'est cette histoire qu'on raconte, qu'on se raconte, suscitant souvent l'incompréhension d'intervenants (notamment en santé) qui peuvent douter de la sincérité du propos devant son caractère «stéréotypé ». Lors d'une demande d'asile, les demandeurs ont à exposer leur parcours, le sentiment de crainte qui a motivé leur départ et les persécutions éventuellement subies. Le représentant de l'Etat chargé de statuer sur la requête, le fait à partir de cette « exposition de soi ». Les demandeurs d'asile doivent établir la preuve de leur condition de victime et prouver leur persécution (DeloryMomberger, 2012). L'histoire est mise en scène dans un objectif précis : il s'agit d'obtenir un statut.

\footnotetext{
${ }^{1}$ Soutenue le 30 novembre 2017 à Lyon II et dont le titre est « Solidarisations, enquête sur les migrants en situation irrégulière et leurs soutiens » sous la direction de Michel Peroni.
} 


\section{Un récit évalué}

Les agents administratifs cherchent à discerner les «bons dossiers », légitimes, s'appuyant sur une réelle persécution, des " mauvais dossiers », illégitimes, parce qu'ils seraient le fait de « fraudeurs » ou parce qu'ils correspondraient en réalité à des départs dus à des difficultés économiques. Il faut être convaincant, tout en sachant qu'il est difficile de connaitre le registre de la pertinence. Selon Estelle d'Halluin, il «s'agit de personnaliser la scène dans la perspective où seule la menace de persécution individuelle est reconnue » (D'Halluin, 2004, p.32). Le récit se doit donc d'être individualisé, rapportant le vécu à une situation personnelle, et précis avec une attention toute particulière au détail, tout en évitant d'insister sur des difficultés économiques qui peuvent disqualifier la demande.

Dans le cadre de la demande d'asile, et que ce soit par les agents de l'OFPRA ou par les juges de la CNDA, le récit est donc évalué. Ce sont la véracité et la sincérité des propos qui sont interrogées. Schématiquement, soit il est jugé crédible, et donc la personne obtient le statut, soit il ne l'est pas, et la personne doit quitter le territoire. Derrière cette évaluation, c'est aussi la migration, l'histoire de vie qui sont examinées. Quels moments, quels évènements valent statut? On est sur le registre de la justification, de la preuve. Il faut prouver ce qu'on avance, pouvoir l'objectiver; alors même que les éléments de preuves font cruellement défaut dans beaucoup de cas. Soit on est jugé victime d'un régime, soit coupable de mensonges et de détournement de procédures.

Vu que ce sont environ deux tiers des demandeurs d'asile qui se voient refuser le statut (après le passage à l'OFPRA et à la CNDA), le récit est généralement évalué de manière «négative ». Souvent pour le migrant, il s'agit alors de reconsidérer une stratégie en vue de rester sur le territoire. Cet instant fait aussi rupture, et l'individu requérant se retrouve dépossédé de son histoire ou plus précisément de celle qu'il vient de raconter et qui avait aussi pour exigence d'être singulière. Les personnes déboutées de la demande d'asile sont avant tout déboutées de leur histoire.

Travail de recadrage : enquêter son histoire 
Un migrant débouté de sa demande d'asile doit ensuite considérer les raisons de l'échec de sa demande et doit nécessairement la recadrer s'il souhaite par exemple prétendre à un titre de séjour particulier ou, et c'est plus rare, pouvoir espérer un réexamen de sa demande d'asile. Ces solutions juridiques sont très complexes et relèvent à chaque fois d'une spécificité : la situation personnelle antérieure, la santé du demandeur ${ }^{2}$ ou encore sa future orientation (comme un mariage)... Il importe alors d'établir les éléments biographiques particuliers permettant d'envisager une régularisation possible sur des critères établis par l'administration, ou sur le registre du « bon sens » quand l'objectif est de demander une décision dérogatoire favorable au préfet.

Il faut donc «passer à autre chose ». Et cela oblige à une activité d'investigation. Qu'est ce qui peut permettre que je reste sur le territoire ? « À quelle porte frapper ? » pour reprendre les termes de Mansour, une personne «sans papiers » régularisée après 6 ans de démarches diverses. À une possibilité donnée correspond un cadre dans lequel l'individu demandeur peut tenter d'être affilié. Mansour a demandé l'asile, puis a tenté un réexamen de l'asile (qui peut être possible à la condition d'avoir de nouveaux éléments). Suite aux refus, il tente de faire les démarches pour que sa mère puisse être française (elle avait vécu en France dans sa jeunesse), puis rencontre les associations d'anciens combattants pour voir si son père qui avait combattu avec la France aurait pu être français. À chaque moment, ce qu'il me raconte diffère mais l'obnubile aussi. Puis l'avocat lui demande d'aller voir un médecin pour avoir un «certificat », au cas où... Même si les chances sont réduites. Finalement il lui dira que la seule solution, «c'est le mariage ». C'est d'ailleurs en se mariant avec une femme française que Mansour obtiendra un titre de séjour. Ainsi importe-t-il pour le requérant, dans un temps très court, souvent dans une situation d'extrême précarité, de faire valoir des éléments de sa vie qui puissent permettre l'accès au séjour, dans un contexte où les possibilités s'amenuisent d'année en année, et où les attentes des professionnels qui viennent en soutien diffèrent les uns des autres.

\footnotetext{
${ }^{2}$ Comme avec la procédure de titre de séjour pour les malades étrangers. La procédure est expliquée ici : https://www.youtube.com/watch?v=SeUR6_BnNGs
} 


\section{Le récit, médiation pour soutien}

Ce récit administratif, à défaut de titre de séjour, devient une sorte de support d'identification susceptible d'évoluer au gré des procédures et des opportunités. Par définition l'activité de soutien est plus complexe d'un point de vue juridique pour les déboutés de l'asile : il faut se saisir de la situation de la personne à partir de ce qu'elle présente sachant qu'il n'est plus possible de se fonder sur la procédure déjà mise en œuvre et par extension sur le récit des persécutions ou menaces de persécutions qui a été formulé dans le cadre de cette même procédure. Dans notre travail doctoral, nous avons investigué et distingué trois espaces ${ }^{3}$ (juridique, politique, social et sanitaire) où les soutiens sont amenés à se rendre solidaires des migrants. Force est de constater que cette injonction à devoir se raconter se retrouve aussi dans ces dispositifs de solidarité, d'entraide ou d'assistance ciblant bien souvent une catégorie de personnes (être demandeur d'asile, être sans ressources...). Pour reprendre Pascale Pichon à propos des SDF, «le récit de soi s'apparente à un plaidoyer plus ou moins adroit en vue de l'obtention de droits et des biens de première nécessité »(Pichon, p.77).

Lors des permanences juridiques, il est demandé aux personnes qui viennent chercher de l'aide de se présenter. Cette présentation va être performante à condition que l'aide demandée entre dans le champ d'expertise et de compétences de l'association ou qu'elle rende sensible celui qui l'écoute. Il s'agit de défendre les droits des étrangers, en conseillant et/ou en accompagnant ces derniers dans la constitution de dossiers de demandes de titres de séjour, et/ou en les accompagnant au contentieux. Dans ce dispositif de la permanence juridique c'est à l'étranger d'amener la preuve de ce qu'il avance. Le juriste, lui, est là pour accompagner, mais ne vient en rien juger de la véracité - ou non - du discours. La supposée neutralité et objectivité du droit permet de ne pas avoir à s'en soucier, et de bénéficier à l'inverse de la confiance des étrangers qui font la démarche de venir dans les permanences, le plus souvent en sachant ce qu'il faut demander. Le jeu est clair. Dans mes observations je n'ai, par exemple, jamais ressenti de malaise, comme cela a pu être le cas dans des collectifs militants, où la place fictionnelle de l'étranger instillait une certaine gêne. La pragmatique qui est à l'œuvre ici est celle d'une aide unidimensionnelle : le problème est juridique, la solution doit l'être également. Dans cette conception participant d'un individualisme rationaliste, il faut dire la vérité aux individus de manière à ce qu'ils puissent s'orienter en connaissance de

\footnotetext{
${ }^{3}$ Qui ont été l'objet de trois parties distinctes.
} 
cause. La permanence, bien souvent, instancie un choix et ce mode opératoire fait des requérants autant d'individus.

Sur le terrain des «mobilisations collectives » auprès des « sans-papiers » et migrants, il n'est pas rare de trouver des «histoires de sans-papiers » dans des témoignages publics. L'histoire est ici mise en scène pour sensibiliser. Selon Olivier Voirol, «la mise en récit procède elle-même d'une distribution des faits et des actions reconnues, fait intervenir des procédures de sélection et d'organisation signifiante "(Voirol, 2005, p.56). Nous avons affaire ici à une activité militante qui consiste à « prélever, compiler, organiser, et constituer des récits par une multitude d'opérations sémantiques, permettant à des phénomènes épars de se relier les uns aux autres »(Voirol, 2005, p.56). Par exemple, avec le développement d'internet, des sites deviennent des plates-formes de témoignages. De même, depuis quelques années le souhait de vouloir faire participer les patients usagers, dans les dispositifs du champ médico-social, se fait souvent sur le registre du témoignage, celui d'un sujet collectif : le migrant, le demandeur d'asile, le malade... L'enjeu est alors d'attirer l'attention publique sur une condition particulière.

Ainsi, les soutiens, par-delà la diversité de leurs domaines et modalités d'intervention, mettent-ils pareillement l'accent sur la narrativité aux fins pratiques de la résolution du problème qui leur importe. Nous faisons alors l'hypothèse que ces injonctions à la mise en récit ont des conséquences sur les individus requérants et obligent à être le «bon » sujet sur les unes et les autres de ces scènes, à tenter d'être le sujet qui correspond à l'objet du dispositif avec lequel il entre en relation.

\section{Se raconter en personne}

Dans le cadre de ce travail de thèse, une personne (en l'occurrence Mansour) a été le sujet et l'objet de ma réflexion. Il ne s'agissait plus d'examiner les manières de venir en soutien aux étrangers, en documentant à chaque fois les effets induits, notamment quant au mode d'appréhension de l'étranger ou du migrant; mais, avec un protocole d'enquête spécifique (entretiens et récits de vie) d'appréhender le migrant directement, sans en passer par le formatage exercé par les divers points d'entrée constitués par les soutiens. Cette 
démarche a été appréhendée comme une tentative d'alternative à la segmentation, participant d'une volonté de retotalisation de l'expérience et de l'existence. Mais ma prétention à vouloir saisir la personne, dans sa dimension la plus totale, a été contrariée par le constat que mon dispositif méthodologique induisait, lui aussi, un format de saisie. Autrement dit, la narration libre proposée à Mansour était aussi un cadre. Pendant 10 ans j'ai côtoyé Mansour, et c'est en partie ce suivi au long cours qui a trouvé in fine son intérêt, l'obtention d'un titre de séjour par Mansour ayant changé la donne, sans mettre fin à la relation, la recalibrant.

Une fois un titre de séjour obtenu suite à un mariage, Mansour a eu l'intention de me raconter la «vraie vie». On peut penser que jusqu'alors, ce qui était occulté, c'est la quotidienneté de l'expérience, sa teneur routinière, celle qui n'est pas en lien avec un problème à résoudre. Derrière la volonté affirmée là par Mansour se joue la réappropriation de son histoire. Comme si le récit avait eu jusqu'alors ses contraintes (réduction, appréciation...) L'enjeu était donc pour lui de reprendre la main : «je prends le stylo », me dit-il. Dire la «vraie vie », c'est aussi sortir de l'exigence d'une histoire particulière et choisir à qui on la raconte. Il n'y a plus d'injonction à l'ordonnancement. Mansour peut «tout mélanger », reprendre le contrôle de l'agencement narratif et sortir des cadres normatifs auxquels il s'était plié.

J'essaie assez vite de voir comment il peut être envisageable, méthodologiquement, que Mansour raconte aussi son histoire de «sans-papiers » ... Quel protocole de travail mettre en place? Je lui propose de commencer par des entretiens hebdomadaires dans un des bureaux du centre de recherche. Et 6 ans après, en me retournant sur cette situation, je comprends d'autant plus que c'est pour s'assurer de la continuité de notre relation qu'il me fait cette proposition, Mansour étant devenu mon ami.

Si jusqu'alors il ne m'avait pas «tout dit », c'est donc, me disais-je, qu'il avait gardé la maîtrise de ce qu'il avait à dire. Qu'est-ce que Mansour aurait désormais à me dire qu'il ne m'avait pas déjà dit ? J'apprends alors qu'il avait de la famille en France et que son grandpère a un patrimoine immobilier dans le sud du pays, ce qu'il ne m'avait jamais dit. C'est moi alors qui mets fin aux entretiens. Je ne m'attendais pas à ce qu'il apporte ces «nouveaux » éléments. Je suis mal à l'aise. C'est surtout le fait qu'il ait de la famille en France qui me surprend. 
Entendant qu'il allait me dire la vérité, me parler de la «vrai vie », je suis dans l'attente d'un nouveau sens. Mais ce qui me déstabilise, au point d'arrêter, c'est qu'il ne se passe pas ce que j'attendais. Ce qui est déstabilisant, ce n'est pas tant le fait qu'il me dise des choses qu'il ne m'avait pas dite, puisque j'étais prévenu ; c'est la nature de ce qui est dévoilé qui me déstabilise. Ces éléments complexifient son récit, rajoutent des protagonistes et me font rentrer un peu plus dans la complication de sa généalogie. Cela fait apparaître combien son récit précédent avait été épuré, schématisé, stylisé. Il avait fait disparaitre des circonstances, des personnages. Maintenant il les rajoute. Pourquoi ? Parce que c'est vrai. Estce aussi par honnêteté ?

Dans mon dernier entretien avec Mansour, j'ai eu également la possibilité de revenir sur le conseil que je lui avais donné, précédent son mariage à la mairie, de ne pas aller à la police. La juriste de l'association déconseillait fortement aux étrangers qui se mariaient de se rendre au commissariat de police s'ils étaient convoqués. Je m'étais fait le porte-voix de ce conseil (j'avais même appelé l'une des juristes pour lui demander confirmation pendant notre entretien). Voici un extrait de l'entretien :

«- Attends Nico, on savait jamais avec les flics, on savait jamais, parce que toi tu fais pas des choses comme ça, mais par contre les flics ils font des choses comme ça. C'est vrai ce que t'a dit la femme de l'association, elle t'a dit : «c'est un piège »

\section{- Ouais}

- Elle t'a dit c'est un piège. Pourtant elle, elle savait, c'est-à-dire elle n'a pas parlé comme ça, Nico, elle savait, parce qu'il y a peut-être des cas qui sont arrivés comme ça. C'est-à-dire tu viens chez nous là, on va te ramasser, normal. Normal. Mais par contre, j'ai regardé gauche et droit, parce que c'était la dernière cartouche, Nico, c'était la dernière carte, parce que cette carte là, c'est soit tu passes, soit tu te casses. Je dis voilà, il faut la jouer, il faut la jouer. Mais j'ai joué avec franchise, avec honnêteté et tout, voilà c'est que je t'ai dit. Même Fatima, elle m'a dit [inaudible, bruit de sirène]

- Fatima t'a dit d'y aller quand même? 
- Ouais, elle m'a dit : « voilà on va... ». Elle m'a dit : « on n'est pas des voleurs, on a rien fait de mal, on essaie de marier ». Elle m'a dit même : «je descends avec toi, mais faut aller. J'aime pas les problèmes avec la police»

- Donc c'était pas prévu qu'elle aille avec toi?

- Hein ?

- C'était pas prévu qu'elle aille avec toi... chez les flics?

- Non, non elle veut pas me lâcher.

- Mais elle avait rendez-vous, elle aussi?

- Non

- D'accord elle y est allé quand même?

- Même elle aussi, ah ouais, elle aussi... J'étais avec toi, qu'elle m'a téléphoné, j'ai dit : «je suis avec Nico », «mais tu sais qu'on a, à une heure et demi, le rendezvous ». J'ai dit : «oui, oui, j'arrive »... [Silence 5 secondes]. T'as pas le choix, parce que si tu pars pas, même... C'est vrai, j'ai rien fait Nico. C'est pas quelque chose que, pourquoi j'ai peur? C'est vrai. Ah franchement. Des fois j'oublie même mon cœur je touche là, parce que vibrations : Mais qu'est-ce que je dois faire ? Il faut aller, il faut aller. Il faut liquider tous ces problèmes là. Parce que, j’ai pensé, si ils voulaient faire, ils savent l'adresse où j'habite, ils peuvent vers minuit, onze heures du soir. Là , Monsieur Haddadi, s’il vous plaît, suivez-moi ». D’accord ? Surtout avec ma taille et tout ! [Rire] Un seul, il me fait comme ça... et hop ! Il faut aller, il faut aller. Je pense même le procureur, il a bien lire tout ce que j'ai fait, il a bien, c'est bon. »

Mansour a beau ne rien ignorer du savoir objectif dont disposent les juristes de l'association appuyés sur la série des cas qu'elles ont eu à connaître, il n'en tire aucunement la conséquence attendue ! C'est la «dernière cartouche », il a épuisé tous les recours juridiques. Et puis la police sait déjà où il habite, donc il se dit que ne pas y aller ne réglerait rien : si les policiers veulent l'arrêter, ils le peuvent. Surtout, il tient à cette honnêteté qui est précisément la sienne. «On n'est pas des voleurs », me dit Mansour. Michel Callon et Vololona Rabeharisoa, rapportant le cas de Gino, un Réunionnais atteint d'une maladie génétique qui refusait, contrairement à son frère Léon, de s'intégrer aux réseaux médicaux et associatifs, et préférant «ne pas savoir » si ses enfants sont porteurs du gène de la maladie, contrevenant 
aux conseils de son entourage, étaient parvenus à cette conclusion : «lorsque Gino déclare qu'il préfère ne pas savoir, il nous livre ce à quoi il tient par-dessus tout et qui forme le cœur de son identité » (Michel Callon et Vololona Rabeharisoa, p. 205). De même, quand Mansour ne suit pas mes conseils, c'est pour faire valoir ce à quoi il tient : son honnêteté. Le refus de Mansour, c'est le refus d'être jugé à l'enseigne des autres.

Cette irréductibilité est celle de la personne, de sa singularité et de son expression, ou pour le dire autrement et en mobilisant les travaux de Ricœur (Ricœur, 1983) celle de l'ipséité, quand les différentes saisies sont toutes construites sur le mode de l'idem de l'identité, de la mêmeté. Il apparaît combien le pouvoir narratif du migrant est plus encore qu'amputé, invalidé, dès lors que la mise en récit que ce dernier ne peut manquer d'opérer est vouée à s'inscrire dans des cadres de validation épistémique et morale qui portent à présupposer que ce qui est dit est vrai. Lors, ce serait bien plutôt par le mensonge que le migrant pourrait reconquérir son ipséité et exister comme une personne à part entière ! Pouvoir tout aussi bien mentir, c'est en effet, pour lui, sortir d'une forme d'assujettissement redoutable puisqu'il est porté à y consentir. C'est au migrant qu'il revient de dire ce qu'il a envie de dire, au moment où il a envie de le dire; aussi est-il capable de raconter des histoires ou du moins de raconter une histoire plus compliquée, comme; celui qui par ailleurs s'est gardé de recourir à l'assistance des soutiens. Un refus que nous avons interprété comme le refus d'être le même que les autres, condition de la solidarité...

Si l'on suit Ricœur, narrer, c'est composer son récit, imaginer. La configuration narrative consiste dans « une opération de mise en intrigue » de l'action racontée et suspend l'action. Cette mise en intrigue correspond à la faculté qu'a le sujet de raconter ses expériences biographiques. Pour Johann Michel et à partir de Paul Ricœur, cette «mise en intrigue [...] provoque une distanciation du réel » (Michel, 2003). «L'histoire d'une vie ne cesse d'être refigurée par toutes les histoires véridiques ou fictives qu'un sujet se raconte sur lui-même » nous dit en effet Paul Ricœur (2003, p.443). Cette capacité narrative est donc indissociable d'une reconnaissance d'une part de fiction dans la narrativité. Mais en aidant les requérants de l'asile à s'acquitter de la tâche de la mise en récit, les soutiens occultent, ou tout du moins invalident cette part fictionnelle immanente à la narrativité. Finalement cette aide est une manière de ne pas habiliter, de ne pas reconnaitre, la compétence narrative. Au vu de notre enquête on peut avancer que la capacité narrative du migrant est alors en partie niée par 
la présupposition même selon laquelle ce qui est dit est vrai. En vis-à-vis et en contrepoint de la suspicion du mensonge des migrants, du côté des institutions étatiques, il y a dans la présomption des soutiens à recueillir la vérité de l'histoire de ces migrants c'est-à-dire dans leur manière de réhabiliter leur récit en le plaçant à leur tour sous le signe de la vérité, un déni de la capacité qu'ont les migrants à se raconter ; un déni symétrique du premier.

\section{Récit, personne et santé mentale}

L'épisode du mensonge de Mansour nous a amené à considérer la singularité de l'étranger, son ipséité, et à faire le constat que les soutiens, dans le cadre de leurs activités, s'appuient au contraire sur une considération de l'autre-même, autrement dit sur la mêmeté de l'étranger. Il nous interroge finalement sur la conditionnalité de l'accueil de l'altérité de l'étranger. Faut-il fatalement descendre en singularité pour accueillir, aider, l'autre ?

C'est ainsi sous le signe de la personne que se déploie aujourd'hui une partie de mon activité à l'Orspere-Samdarra ${ }^{4}$. Engagé dans un processus d'enquête collective pour œuvrer à la problématisation conjointe de ce que la question de la santé mentale fait au migrant, et de la question que pose le migrant à la santé mentale ; et ce faisant, élargir le concernement des professionnels, ouvrir à de nouvelles pratiques qui, tant que faire se peut, prennent en compte la personne migrante, même saisie du côté de la vie quelconque et ordinaire (Agamben, 1990).

Cette perspective nous met aux prises avec des intervenants en santé mentale, particulièrement d'inspiration analytique, qui ont la prétention à appréhender la personne dans sa dimension totale. Mais quand justement le matériau clinique se base aussi sur le récit de soi, le soignant se retrouve en difficulté, notamment face aux stratégies mises en place par les migrants. La prise en compte de la dimension traumatique de l'expérience est paradigmatique de cet embarras. Alors que le traumatisme devient le ressort majeur de preuve de véridiction conditionnant l'asile (Fassin, 2007, p.328), les psychistes peuvent être appelés à venir certifier sur un registre d'objectivation nosographique les conséquences sur la santé mentale de cette

\footnotetext{
${ }^{4}$ Observatoire-Ressource sur des thématiques qui lient problématiques santé mentale et questions sociales (précarités, vulnérabilités, migrations...), hébergé au CH Le Vinatier à Lyon.
} 
expérience, parfois en inadéquation avec la perspective thérapeutique où la mise en récit se pense comme performative. Et si les «nouvelles» pratiques cliniques (de réhabilitation, de rétablissement...) s'émancipent du récit comme matériau clinique en centrant l'exercice sur le «projet »d'aller mieux, et donc s'affranchissent de l'entrée victimaire ; elles butent sur la précarité de l'inscription sociale de la personne.

Force est de tenir compte de ce qu'existent des épreuves d'hospitalité, notamment à l'hôpital, incarnation instituée de l'hospitalité. C'est d'ailleurs ce que nous interrogeons dans le cadre d'une recherche sur l'interprétariat en santé ${ }^{5}$. Pour comprendre celui ou celle qui ne parle pas la langue, il faut parfois faire appel à un interprète ou à un médiateur. Jacques Derrida et Anne Dufourmontelle affirme justement que «la question de l'hospitalité commence là : devons-nous demander à l'étranger de nous comprendre, de parler notre langue, à tous les sens de ce terme, dans toutes ses extensions possibles, avant et afin de pouvoir l'accueillir chez nous ? S'il parlait déjà notre langue, avec tout ce que cela implique, si nous partagions déjà tout ce qui se partage avec une langue, l'étranger serait-il encore un étranger et pourrait-on parler à son sujet d'asile ou d'hospitalité ?» (Derrida, Dufourmontelle, 2007, p.21). L'interprète est alors cette figure qui peut permettre aux altérités de co-exister dans la relation. En pratique, quels sont ajustements pour qu'il y ait justement une relation ? Quelle pragmatique de l'hospitalité ? À quoi peut engager un accueil dont le format de saisie de son public dérogerait à l'ordre de la mêmeté ? Il importe alors de documenter le travail de front, clinique ou d'accompagnement des migrants par les professionnels de santé mentale effectué dans le cadre du colloque singulier ${ }^{6}$.La caractérisation de l'altérité, que ce soit en termes pathologiques, sociaux, ou culturels, n'opère plus forcément en vue de son effacement mais fait bien plutôt l'objet d'une réappropriation par les personnes concernées.

\section{Bibliographie}

Agamben Giorgio (1990), La communauté qui vient. Théorie de la singularité quelconque, Paris, Le Seuil.

\footnotetext{
${ }^{5}$ Dans le cadre de la recherche ANR Remilas portée par Véronique Traverso (ICAR, ENS Lyon).

${ }^{6}$ Les linguistes du laboratoire ICAR utilisent comme matériau d'enquête des vidéos de consultation en santé.
} 
Belkis Dominique, Franguiadakis Spyros (2008), «Devenir réfugié : expérience de parcours, épreuve identitaire et récit biographique », actes du colloque international Terrains d'asile. Corps, espaces, politiques, EHESS, Paris.

Callon Michel, Rabeharisoa Vololona (1999), «La leçon d'humanité de Gino », Dans Réseaux, volume 17, n95, 1999. Sciences, malades et espace public, p. 197-233.

Chappe Vincent-Arnaud (2010), « La qualification juridique est-elle soluble dans le militantisme ? Tensions et paradoxes au sein de la permanence juridique d'une association antiraciste », Droit et société, 2010/3, n 76, p. 543-567.

Delory-Momberger Christine (2012), « La langue coupée. Quand le récit doit faire preuve ». In A. Brossat \& M. Déotte (dir.). Corps subalternes. Migrations, expériences, récits. Paris : L'Harmattan, p. 159-172.

Derrida Jacques, Dufourmontelle Anne (1997), De l'hospitalité, Paris, Calmann Levy.

Dodier Nicolas, Rabeharisoa, Vololona (2006), « Les transformations croisées du monde « psy » et des discours du social », Politix, 2006/1, n 73, p. 9-22.

Fassin Didier (2004), Des maux indicibles, sociologie des lieux d'écoute, Paris, La Découverte.

Fassin Didier, Rechtman Richard (2007), L'empire du traumatisme, enquête sur la condition de victime, Paris, Flammarion.

Franguiadakis Spyros, Jaillardon Édith et Belkis Dominique (2004), En quête d'asile. Aide associative et accès au $(x)$ droit(s), Paris, LGDJ, (Droit et société).

d'Halluin Estelle (2004), «Comment produire un discours légitime ? », Plein droit, $\mathrm{n}^{\circ} 63$, décembre 2004, p. 30-33.

Michel Johann (2003), « Narrativité, narration, narratologie : du concept ricœurien d'identité narrative aux sciences sociales », Revue européenne des sciences sociales, XLI-125/2003, p. $7-14$.

Pichon Pascale (2014), «Vulnérabilité narrative et expressions biographiques depuis l'expérience sans domicile fixe » in Axelle Brodiez-Dolino, Isabelle von Bueltzingsloewen, Benoît Eyraud, Christian Laval et Bertrand Ravon (dir.), vulnérabilités sanitaires et sociales, PUR, Rennes.

Ricœur Paul (1983), Temps et récits III, Le temps raconté, éditions du Seuil.

Voirol Olivier (2005), « Le travail normatif du narratif. Les enjeux de reconnaissance dans le récit médiatique », Réseaux 2005/4, n 132, p.51-71. 
